\title{
A New Approach of Blind Image Deconvolution Method without Artifact along the Blurring Paths
}

\author{
W. F. Al Maki, T. Shimahashi, T. Kitagawa, and S. Sugimoto \\ Dept. of Electrical and Electronic Engineering, Ritsumeikan University \\ 1-1-1, Noji-Higashi, Kusatsu City, Shiga 525-8577, Japan \\ Tel: +81-(0)77-561-2673, Fax: +81-(0)77-561-2663 \\ E-mail: sugimoto@se.ritsumei.ac.jp
}

\begin{abstract}
In this paper, we consider a blind image deconvolution method for linear motion blurred images. Point spread function (PSF) of the linear motion blur has two parameters, i.e., motion length and motion direction. The parameters are estimated using the modified discrete Radon transform and cepstral analysis. The estimated PSF parameters are then used in the image deconvolution process. To restore the blurred images, we first model the 2-D degradation process into 1-D form along the blurring path. Therefore, the new 1-D blurred image model is constructed for each row of the blurred image in the $2-\mathrm{D}$ degradation process. The corresponding PSF matrix is then inverted to get the true image. Finally, experimental results show our proposed idea.
\end{abstract}

\section{Introduction}

A linear motion blur is a common degradation which is caused by relative motion between the camera and the captured object. In order to reconstruct the true image from the linear motion blurred image, the point spread function (PSF) must be known. However, the PSF is unknown in practical situation. For linear motion blur case, to obtain the PSF, it is sufficient to estimate its parameters, namely motion direction and motion length. The accuracy of parameter estimation of the PSF plays important role in the blind image deconvolution. Many researchers have involved in this area, but most of them assume that the motion direction is horizontal. In [1] and [2], bispectrum is used in blur identification. PSF parameters identification using power cepstrum is presented in [3], [4], and [5].

In this paper, we propose an algorithm of blind image deconvolution without artifact. We apply the modified discrete Radon transform as line detection algorithm and the 2-D cepstral analysis for estimating the PSF parameters. The discrete Radon transform used in our works does not employ a complicated algorithm as shown in the conventional discrete Radon transform [8] to fit the pixels along the reference lines.

To increase accuracy of motion direction estimation using the Radon transform, we consider the modification to the discrete Radon transform and a windowing method to the blurred image. The modified Radon transform can solve the inherent problem in the conventional Radon transform, i.e. the number of pixels projected on a line is not necessarily the same for different directions and/or coordinates of a direction. Therefore, the summation of all coordinates along the line in the s-axis may not act in a similar statistical base for different parameters $\rho$ and/or $\theta[9]$.

As either the modified Radon transform or the 2-D cepstral analysis has their own capabilities for certain value of the estimated motion length, to determine the better method between them, the estimated motion length is used as a criterion. The motion length itself is estimated using the 2-D cepstral analysis.

The true image is obtained via spatial domain-based inverse filtering using the estimated PSF parameters. First, we reformulate the 2-D model of degradation process into its 1-D model. Therefore, the new model is expressed for each row of the blurred image. By using this model, degradation process along the blurring path can be described by a lower triangular matrix. In this 1-D degradation model, the pixels values along the blurring path are obtained by applying bilinear interpolation. The true image is obtained by applying matrix inversion.

\section{Motion Blured Image and PSF Model}

The linear motion blurred image $g(x, y)$ can be modeled as an output of 2-D convolution process between the true image $f(x, y)$ and the PSF $h(x, y)$. In the noiseless motion blurred image case, the model is given by

$$
g(x, y)=f(x, y) \circledast h(x, y)
$$

The PSF $h(x, y)$ in the spatial domain can be modeled as $[6]$

$$
h(x, y)= \begin{cases}1 / L & \text { if } 0 \leq|x| \leq L \cos \theta ; y=L \sin \theta \\ 0 & \text { otherwise }\end{cases}
$$


In this paper, we work in the frequency domain. Since the convolution in the spatial domain is equal to multiplication in the frequency domain, then in the frequency domain (1) can be written as

$$
G\left(\omega_{x}, \omega_{y}\right)=F\left(\omega_{x}, \omega_{y}\right) H\left(\omega_{x}, \omega_{y}\right)
$$

where $G\left(\omega_{x}, \omega_{y}\right), F\left(\omega_{x}, \omega_{y}\right)$, and $H\left(\omega_{x}, \omega_{y}\right)$ are the frequency responses of blurred image, true image and PSF, respectively.

In (3), $H\left(\omega_{x}, \omega_{y}\right)$ is expressed as

$$
H\left(\omega_{x}, \omega_{y}\right)=\operatorname{sinc}\left(\pi L\left(\omega_{x} \cos \theta+\omega_{y} \sin \theta\right)\right)
$$

where $\operatorname{sinc}(x)=\frac{\sin x}{x}$

The sinc structure, shown in (4), appears as parallel dark lines whose width and direction correspond to the motion length $(L)$ and the motion direction $(\theta)$, respectively. Since the spectrum of linear motion blurred image is biased in the direction of the blur (anisotropy), the parallel dark lines are also available in the spectrum of the blurred image. Therefore, $L$ and $\theta$ can be obtained directly from the blurred image.

\section{Estimation of PSF Parameters}

In this section, we propose the algorithms to estimate linear motion blur PSF parameters.

\subsection{Motion Length Estimation}

Frequency response of linear motion blur is characterized by zero crossings that occur periodically along the lines perpendicular to the direction of motion blur. The frequency zero crossing happens at $\mu=m / L$, where $\mu=\zeta_{1} \cos \theta+\zeta_{2} \sin \theta$ and $m=0, \pm 1, \pm 2, \pm 3, \ldots$. Cepstral analysis can be used to estimate these periodic zeros.

In this paper, two-dimensional power cepstrum of $g(x, y)$ is used. The power cepstrum is given by

$$
\hat{g}(x, y)=\hat{f}(x, y)+\hat{h}(x, y)
$$

In (5), $\hat{g}(x, y)$ is expressed as

$$
\hat{g}(x, y)=\mathcal{F}^{-1} \log \left\{\left|G\left(w_{x}, w_{y}\right)\right|\right\}
$$

where $\mathcal{F}^{-1}$ denotes the inverse Fourier transform operator. $\hat{f}(x, y)$ and $\hat{h}(x, y)$ are expressed in the similar way as shown in (6).

By applying the cepstral analysis, a large negative peak at a distance $L$ from the origin will appear at $\hat{h}$ [4]. As sinc structure whose periodicity contains information about motion length is also maintained in the Fourier spectrum of the blurred image, the large negative peak also occurs when cepstral analysis is applied to it. The motion length is then obtained by calculating the distance of the large negative peaks from the origin.

In order to get better results of estimation of the PSF parameters, a Gaussian window is used for masking before estimating the motion length. The coefficients of the Gaussian window is computed by

$$
w(m, n)=\frac{1}{2 \pi \sigma^{2}} e^{-\frac{1}{2}\left(\frac{m^{2}+n^{2}}{\sigma^{2}}\right)}
$$

\subsection{Motion Direction Estimation}

Motion direction is estimated using the modified Radon transform and cepstral analysis. Motion direction estimation using cepstral analysis is performed by calculating the slope of a line connecting the origin to the large negative value of the cepstrum of the blurred image. We use both the cepstral analysis and the modified Radon transform in motion direction estimation. In [9], the modified Radon transform is utilized as a method for direction estimation. The modified Radon transform is applied to standard texture images where the texture can be one-directional, multi-directional, mixed, or isotropic. Experimental results show that the modified Radon transfom significantly outperform the conventional Radon transform both in noiseless and noisy image cases. In our works, the task is easier since the lines that appear in the spectrum of the blurred image are in one direction.

The sharp edges of the image will cause additional lines in the spectral domain. The Radon transform will detect these edges. To avoid this effect, the boundaries of the image are smoothened out using a Gaussian window before estimating the motion direction. Since the values of the Gaussian windowed image will decay towards the image boundary, the edge effects disappear. Therefore, the blurred image is smoothened before PSF parameters estimation methods are applied.

The Radon transform is given by [7]

$g(\rho, \theta)=\int_{-\infty}^{\infty} \int_{-\infty}^{\infty} f(x, y) \delta(x \cos \theta+y \sin \theta-\rho) \mathrm{d} x \mathrm{~d} y$

Equation (8) can also be written as

$$
g(\rho, \theta)=\int_{-\infty}^{\infty} f(\rho \cos \theta-s \sin \theta, \rho \sin \theta+s \cos \theta) \mathrm{d} s
$$

In (9), $\rho$ and $s$ can be calculated from $x, y$, and $\theta$ using $\rho=x \cos \theta+y \sin \theta$ and the following equation, respectively:

$$
s=\frac{\rho \cos \theta-x}{\sin \theta}=\frac{y-\rho \sin \theta}{\cos \theta}=y \cos \theta-x \sin \theta
$$

In order to apply the Radon transform on a 2-D digital image $z(m, n)$ of size $M \times N$, we first sample continuous variables [9]:

$$
\begin{array}{ll}
x=x_{m}=x_{\min }+m . \Delta x, & m=0,1, \cdots, M-1 \\
y=y_{n}=y_{\min }+n \cdot \Delta y, & n=0,1, \cdots, N-1 \\
\theta=\theta_{t}=\theta_{\min }+t . \Delta \theta, & t=0,1, \cdots, T-1 \\
\rho=\rho_{r}=\rho_{\min }+r . \Delta \rho, & r=0,1, \cdots, P_{\theta}-1 \\
s=s_{k}=s_{\min }+k . \Delta s, & k=0,1, \cdots, S_{\rho, \theta}-1
\end{array}
$$


where

$$
\begin{aligned}
& x_{\min }=-x_{\max }=-(M-1) / 2, \\
& y_{\min }=-y_{\max }=-(N-1) / 2, \\
& \rho_{\min }=-\rho_{\max }=-\frac{\left(P_{\theta}-1\right)}{2} \Delta \rho, \\
& s_{\min }=-s_{\max }=-\frac{\left(S_{\rho, \theta}-1\right)}{2} \Delta s .
\end{aligned}
$$

$\Delta \theta$ is set to $\pi / T$ in order to let $\theta \operatorname{span} \pi . \Delta \rho, \theta_{\text {min }}$, $\Delta x, T$, and $\Delta y$ are set to $1,0,1,180$, and 1 , respectively. The values of parameters $\Delta s, P_{\theta}$, and $S_{\rho, \theta}$ depend on $\rho$ and/or $\theta$. They should be set precisely to let all pixels of the image be utilized in the Radon transform for any angle $\theta$. Using (10) and (11), $\Delta s$ and $P_{\theta}$ can be approximated as [9]

$$
\begin{gathered}
\Delta s= \begin{cases}\frac{1}{\left|\cos \theta_{t}\right|}, & \sin \theta_{t} \leq \sin \frac{\pi}{4}=\frac{1}{\sqrt{2}} \\
\frac{1}{\sin \theta_{t}}, & \sin \theta_{t}>\sin \frac{\pi}{4}=\frac{1}{\sqrt{2}}\end{cases} \\
P_{\theta} \approx\lceil M \Delta s\rceil+1-(\lceil M \Delta s\rceil \bmod 2)
\end{gathered}
$$

where $\lceil$.$\rceil denotes the ceiling operation, and P_{\theta}$ is adjusted to take an odd integer value.

The value of $S_{\rho, \theta}$ depends on both $\rho$ and $\theta . S_{\rho, \theta}$ will be arithmetically decreased in a series afterwards, depending on both $\rho$ and $\theta$, if it is fixed and equal to $M$ for some values of $\rho$. In this paper, $S_{\rho, \theta}$ is approximated as follows [9]

$$
S_{\rho, \theta}= \begin{cases}M, & \left|\rho_{r}\right| \leq \rho_{\text {const }} \\ M-\left[\left(\left|\rho_{r}-\rho_{\text {const }}\right|\right) \cdot d\right], & \left|\rho_{r}\right|>\rho_{\text {const }}\end{cases}
$$

In (15), $\rho_{\text {const }}$ and $d$ are [9]

$$
\begin{aligned}
\rho_{\text {const }} & =\left[\frac{M-1}{1}|| \cos \theta_{t}\left|-\sin \theta_{t}\right|\right], \\
d & =\frac{2(\alpha M-\beta)}{\alpha(\alpha-1)} \\
\alpha & =\left(\frac{P_{\theta}-1}{2}\right)-\rho_{\text {const }}+1 \\
\beta & =\frac{M^{2}}{2}-M \rho_{\text {const }}
\end{aligned}
$$

where [.] denotes the rounding operation.

In the approximation of $S_{\rho, \theta}$, each pixel is assumed to be assigned to its nearest reference line. Since the pixels are not always exactly on the reference lines, rounding operation is applied here for simplicity. Therefore, by defining $x_{k}^{\prime}$ and $y_{k}^{\prime}$ as [9]

$$
\begin{aligned}
x_{k}^{\prime} & =\left[\rho_{r} \cos \theta_{t}-s_{k} \sin \theta_{t}-x_{m i n}\right], \\
y_{k}^{\prime} & =\left[\rho_{r} \sin \theta_{t}+s_{k} \cos \theta_{t}-y_{\text {min }}\right],
\end{aligned}
$$

the discrete Radon transform of a 2-D digital image $g(m, n)$ can be approximated from (9) as

$$
g\left(\rho_{r}, \theta_{t}\right) \approx \Delta s \sum_{k=0}^{S_{\rho, \theta}-1} g\left(x_{k}^{\prime}, y_{k}^{\prime}\right)
$$

In this paper, a modification to the discrete Radon transform expressed in (19) is considered. First, we define a set $\Psi^{r, t}$, which is slightly different from [9], as follows.

$\Psi^{r, t}=\left\{\left(g\left(x_{i}^{\prime}, y_{i}^{\prime}\right)\right): \rho=\rho_{r}\right.$ and $\left.\theta=\theta_{t}, i=0,1, \ldots, S_{\rho, \theta}-1\right\}$

By using (20), (19) is rewritten as

$$
g\left(\rho_{r}, \theta_{t}\right) \approx \Delta s \sum_{k=0}^{S_{\rho, \theta}-1} \Phi_{k}^{r, t}
$$

where $\Phi_{k}^{r, t}$ is the $k^{t h}$ element of the set $\Psi^{r, t}$.

Eq. (21) is then rewritten as [9]

$$
g\left(\rho_{r}, \theta_{t}\right) \approx \Delta s S_{\rho, \theta} \operatorname{mean}\left(\Psi_{k}^{r, t}\right)
$$

From (22), the modified discrete Radon transform is defined as [9]

$$
g\left(\rho_{r}, \theta_{t}\right) \equiv \Delta s \operatorname{mean}\left(\Psi_{k}^{r, t}\right)
$$

Since the sets $\Psi^{r, t}$ have different number of elements, a more stabilize statistical behavior as compared to the conventional Radon transform is obtained, if sample mean is used as shown in (23), instead of summation in (19). Thus, a better performance for direction estimation is achieved [9].

In order to apply the discrete Radon transform, Fourier spectrum of blurred image is treated as an image. To find direction of the lines which appear in the spectrum, the Radon transform is calculated for all directions with $\theta_{t}=\left[0, \pi-\frac{\pi}{180}\right]$. We compute the variance of the results for each direction, and then form the variance array $V_{R}$ as

$$
V_{R}\left(\theta_{t}\right)=\operatorname{Var}_{\rho_{r}}\left[g\left(\rho_{r}, \theta_{t}\right)\right], \quad \theta_{t} \in\left[0, \pi-\frac{\pi}{180}\right]
$$

The motion direction is obtained by finding the maximum value of variance array.

\section{Image Deconvolution}

In this paper, linear spatially-invariant degradation process is considered. Therefore, the $M \times N$ blurred image is expressed as

$$
g(m, n)=\sum_{p=-P}^{P} \sum_{q=-Q}^{Q} h_{p, q} f(m-p, n-q)
$$


In our work, we reformulate (25) to get 1-D degradation process. For linear motion blurred image, the blurred image at $m^{\text {th }}$ row is defined as

$$
\begin{aligned}
g_{m}(n) & =\sum_{q=-Q}^{Q} h_{q} f_{m}(n-q) \\
& =\sum_{l=-L / 2}^{L / 2} \frac{1}{L} f_{m}(n-l)
\end{aligned}
$$

Equation (27) can be written as

$$
g_{m}(n)=\sum_{l=0}^{L-1} \frac{1}{L} f_{m}(n-l)
$$

The corresponding matrix form of the aforesaid formula is given by

$$
G_{m}=H F_{m}
$$

where $G_{m} \in \Re^{N \times 1}, H \in \Re^{N \times N}$, and $F_{m} \in \Re^{N \times 1}$ are defined by the following matrices

$$
\begin{gathered}
G_{m}=\left[\begin{array}{c}
g(m, 1) \\
g(m, 2) \\
\vdots \\
\vdots \\
g(m, N)
\end{array}\right] \quad F_{m}=\left[\begin{array}{c}
f(m, 1) \\
f(m, 2) \\
\vdots \\
\vdots \\
f(m, N)
\end{array}\right] \\
H=\left[\begin{array}{cccccc}
h_{0} & & & & 0 \\
h_{1} & \ddots & & & \\
\vdots & \ddots & \ddots & & \\
h_{L-1} & \cdots & \cdots & h_{0} & & \\
& \ddots & \ddots & \ddots & \ddots & \\
0 & & h_{L-1} & \ldots & \ldots & h_{0}
\end{array}\right]
\end{gathered}
$$

From (29), the 1-D estimated true image is obtained by applying (32) to each row $(m=1,2, \ldots M)$.

$$
\hat{F}_{m}=H^{-1} G_{m}
$$

$H^{-1}$ in (32) is computed by applying elementary row operations. The restored image $\hat{F}$ is then defined as

$$
\hat{F}=\left[\begin{array}{c}
\hat{F}_{1}^{\mathrm{T}} \\
\hat{F}_{2}^{\mathrm{T}} \\
\vdots \\
\vdots \\
\hat{F}_{M}^{\mathrm{T}}
\end{array}\right]
$$

where $\hat{F}$ is a $M \times N$ matrix.
As for the motion blurred image with $0<\theta<\frac{\pi}{2}$, we redefine (25) as

$$
z(i, j)=\sum_{p=-P}^{P} \sum_{q=-Q}^{Q} h_{p, q} u(i-p, j-q)
$$

where $u(i, j)$ is the bilinear-interpolated pixel obtained from the original image $F(m, n)$ pixels along the blurring path at the motion direction. Therefore, the size of $U$ is not equal to that of $F$ anymore. If the size of $\mathrm{F}$ is $M \times N$, then the size of $U$ is $I \times J$. $I$ is given by

$$
I=M+\lceil(\tan \theta \times(N-1))\rceil
$$

where $\lceil\cdot\rceil$ denotes the ceiling operation.

The value of $\mathrm{J}$ is different for different size of the image as shown below.

For $N>M$ :

$$
J=\left\lfloor\frac{1}{\cos \theta}\{(N-1)-Y\}\right\rfloor+1
$$

For $N \leq M$ :

$$
J=\left\lfloor\frac{1}{\sin \theta}\{(N-1) \times \tan \theta\}\right\rfloor+1
$$

where $\lfloor\cdot\rfloor$ denotes the flooring operation.

In (36), $\mathrm{Y}$ is given by

$$
Y=\frac{1}{\tan \theta}\{((N-1) \times \tan \theta)-(I-1)\}
$$

By employing (35) - (38), we can treat the degradation process which happens at $0<\theta<\frac{\pi}{2}$ as the degradation process in horizontal direction. From (34), the blurred image at $i^{\text {th }}$ row is defined as

$$
z_{i}(j)=\sum_{l=0}^{L-1} \frac{1}{L} u_{i}(j-l)
$$

In matrix representation, (39) is written as

$$
Z_{i}=H U_{i}
$$

where each $Z_{i} \in \Re^{J \times 1}, U_{i} \in \Re^{J \times 1}$, and $H \in \Re^{J \times J}$ is defined in the same way as shown in (31).

Then, the 1-D deconvolved image is given by

$$
\hat{U}_{i}=H^{-1} Z_{i}
$$

The restored image obtained by the aforesaid formula is a vector matrix. As the desired image must have a full matrix form, (41) is applied to each row of $Z$, i.e. $i=1,2, \ldots I$. Therefore, a restored image along the blurring path can be obtained by as shown in (42).

$$
\hat{U}=\left[\begin{array}{c}
\hat{U}_{1}^{\mathrm{T}} \\
\hat{U}_{2}^{\mathrm{T}} \\
\vdots \\
\vdots \\
\hat{U}_{I}^{\mathrm{T}}
\end{array}\right]
$$


Because each element of $\hat{U}$ is the bilinear-interpolated pixel along the motion direction, $\hat{U}$ is reinterpolated using the bilinear interpolation to get the true restored image.

As for the motion blurred image with $\frac{\pi}{2}<\theta<\pi$, we first reformulate (34) as

$$
z_{f l i p}(i, j)=\sum_{p=-P}^{P} \sum_{q=-Q}^{Q} h_{p, q} u_{f l i p}(i-p, j-q)
$$

The matrix representation of (43) is

$$
Z_{\text {flip }}=H U_{f l i p}
$$

where $U_{\text {flip }}$ is obtained by performing left-right flipping operation to $F$ and then interpolating $F$ along the blurring path (motion direction) . Therefore, the $Z_{\text {flip }}$ matrix, whose elements are $z_{f l i p}(i, j)$, is a left-right flipped matrix. In the simulation, we also consider that $Z_{\text {flip }}$ matrix is obtained from left-right flipping operation on $Z$ matrix. The matrix size of $Z_{\text {flip }}$ is $I \times J$. I and $J$ are defined as

$$
I=M+\lceil(\tan \psi \times(N-1))\rceil
$$

For $N \leq M$ :

$$
J=\left\lfloor\frac{1}{\sin \psi}\{(N-1) \times \tan \psi\}\right\rfloor+1
$$

For $N>M$ :

$$
J=\left\lfloor\frac{1}{\cos \psi}\{(N-1)-Y\}\right\rfloor+1
$$

where

$$
Y=\frac{1}{\tan \psi}\{((N-1) \times \tan \psi)-(I-1)\}
$$

In (45) - (48), $\psi=\pi-\theta$.

By using the same way shown in (39) - (42), we get the restored image. However, this image is a left-right flipped matrix. The final restored image is obtained by applying left-right flipping operation on it.

\section{$5 \quad$ Simulation and Results}

Our experiments are performed by using computer with specifications: Intel Pentium M $1.86 \mathrm{GHz}$ and 1 GB RAM. The software used in the simulation is Matlab 6.5.1. To approach practical situation, we employ RGB images. Artificially blurred image is obtained by blurring simulation with angles varying from 0 to $\pi-\frac{\pi}{180}$ and length varying from 1-79 pixels. Before estimating the parameters of the PSF, the blurred image is windowed using a Gaussian window. In Gaussian windowing, the $\sigma$ parameter can be chosen arbitrarily only to smoothen edges of the image. In our experiments, we use $\sigma=5$. Fig. 1, Fig. 2, Fig. 3, and Fig. 4 show the original image, the blurred image, the Gaussianwindowed blurred image, and the restored image (deconvolved image) respectively

In this paper, the motion length $(L)$ is estimated using only the 2-D cepstral analysis. However, in motion direction estimation $(\theta)$, we use two methods. As the cepstral analysis and the modified Radon transform has different capabilities for certain value of motion length, the two methods are used together. For $L<14$ pixels, the cepstral analysis gives better motion direction estimation than the Radon transform does. The maximum error when we use the cepstral analysis is 3 degrees, but it becomes bigger if we use the modified Radon transform. For $L \geq 14$ pixels, the modified Radon transform can give better estimation than the cepstral analysis. Therefore, the estimated motion length obtained by the cepstral analysis is used to determine the better method to estimate the motion direction. If $L<14$ pixels, then the cepstral analysis is used to estimate the motion direction. If $L \geq 14$ pixels, the motion direction is estimated using the modified discrete Radon transform.

Table 1: Performance of Estimation and Image Deconvolution Method

\begin{tabular}{|c|c|c|c|c|}
\hline $\mathrm{L}$ & $\theta$ & $\hat{L}$ & $\hat{\theta}$ & $M S E$ \\
\hline \hline 13 & 23 & 15 & 22 & 0.0535 \\
\hline 15 & 43 & 16 & 43 & 0.0541 \\
\hline 24 & 136 & 24 & 138 & 0.0380 \\
\hline 48 & 18 & 49 & 17 & 0.0467 \\
\hline 53 & 27 & 54 & 27 & 0.0316 \\
\hline 63 & 5 & 64 & 4 & 0.0283 \\
\hline
\end{tabular}

The performances of the method of PSF parameters estimation and image deconvolution are shown in Table 1. In Table 1, MSE (mean square error) is computed using

$$
M S E=\frac{1}{M N} \sum_{m=0}^{M-1} \sum_{n=0}^{N-1}[\hat{f}(m, n)-f(m, n)]^{2}
$$

where $\hat{f}(m, n)$ and $f(m, n)$ denote the restored image and the original image, respectively.

\section{Conclusions}

PSF parameters estimation using the modified discrete Radon transform and the 2-D cepstral analysis has been demonstrated. Simulation results show the capability of the methods for restoring blurred image. Our method can be applied to any image size, any motion direction, and any motion length. By applying two methods which have their own capability, the accuracy of motion direction estimation using our method is better than by just applying one of the methods. In future works, we will consider a method to make the restored 


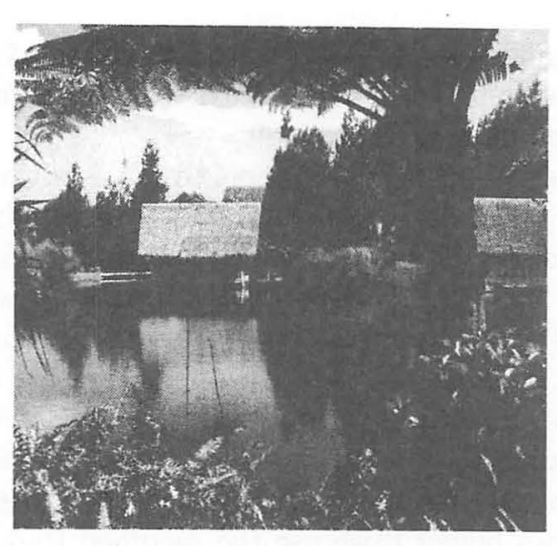

Fig. 1: Original Image

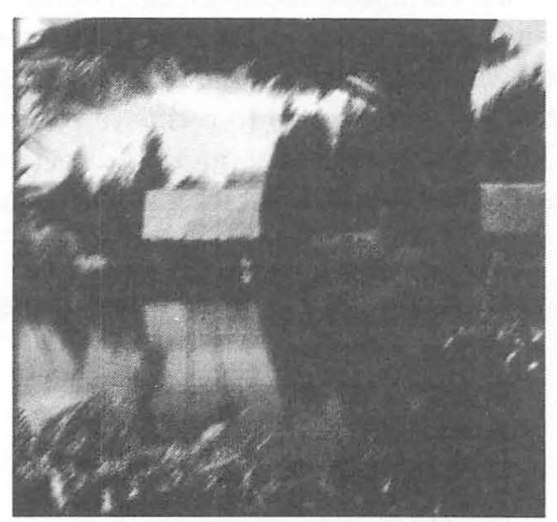

Fig. 2: Motion Blurred Image $\left(L=15\right.$ pixels, $\left.\theta=43^{\circ}\right)$

image becomes clearer, and extend the problem to noisy blurred image case.

\section{References}

[1] M. M. Chang, A. M. Tekalp, and A. T. Erdem: Blur identification using the bispectrum, IEEE Trans. Signal Process. 39(10), pp. 2323-2325, 1991.

[2] C. Mayntz, T. Aach, and D. Kunz: Blur identification using a spectral inertia tensor and spectral zeros Proc. IEEE International Conference on Image processing(ICIP), 1999.

[3] M. Cannon: Blind deconvolution of spatially invariant image blurs with phase, IEEE Trans. Acoust. Speech Signal Process. 24(1), pp. 56-63, 1976.

[4] J. Biemond, R. L. Lagendijk, and R. M. Mersereau: Iterative methods for image deblurring, Proc. of the IEEE, pp. 856-883, 1990.

[5] Ioannis M. Rekleities: Optical flow recognition from the power spectrum of a single blurred image,

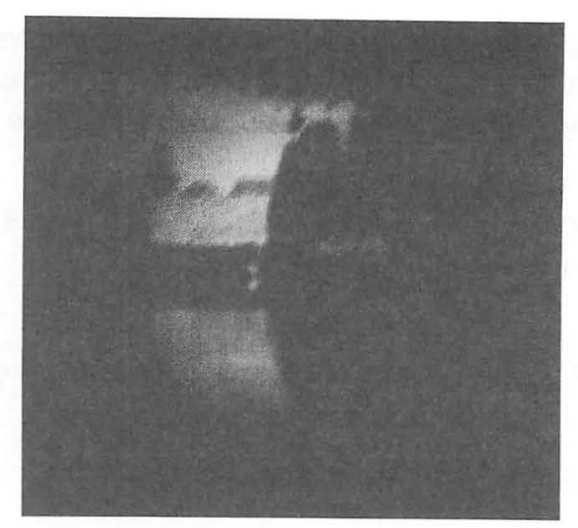

Fig. 3: Gaussian-windowed Blurred Image

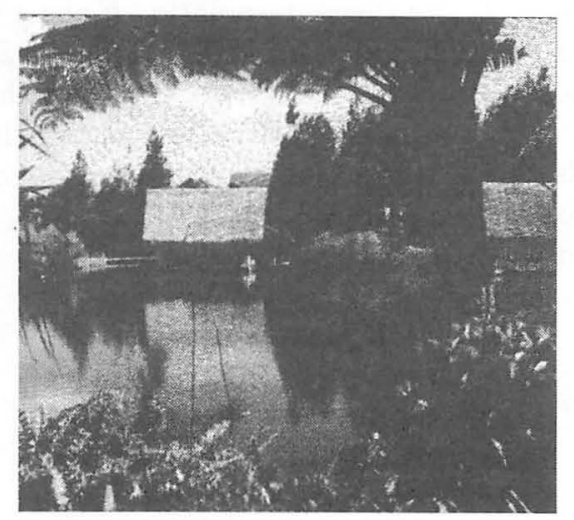

Fig. 4: Restored Image using $\hat{L}=16$ pixels and $\hat{\theta}=43^{\circ}$

IEEE International Conference on Image processing(ICIP), 1996.

[6] R. Lokhande, K. V. Arya, and P. Gupta: Identification of parameters and restoration of motion blurred images, Proc. of ACM Symposium on Applied Computing, pp. 301-315, 2006.

[7] A. K. Jain: Fundamentals of Digital Image Processing, Prentice Hall, New Jersey, 1989.

[8] P. Toft: The Radon Transform: Theory and Implementation, Ph.D thesis, Technical University of Denmark, 1996.

[9] M. R. Hajazi, G. Shevlyakov, and Y. S. Ho: Modified discrete Radon transforms and their application to rotation-invariant image analysis, IEEE 8th Workshop on Multimedia Signal Processing, 2006. 